\title{
Molecular Size Effect on Curing Process for Epoxy and Amine Mixture
}

\author{
Satoru Yамамото ${ }^{* \dagger}$ and Keiji TANAKA ${ }^{*, * *, * * *, * * * *, \dagger}$ \\ "Center for Polymer Interface and Molecular Adhesion Science, Kyushu University, Fukuoka 819-0395, Japan \\ ${ }^{* *}$ Department of Applied Chemistry, Kyushu University, Fukuoka 819-0395, Japan \\ ${ }^{* * *}$ Department of Automotive Science, Kyushu University, Fukuoka 819-0395, Japan \\ ${ }^{* * * *}$ International Institute for Carbon-Neutral Energy Research (WPI-I2CNER), Kyushu University, Fukuoka 819-0395, Japan \\ (Received : September 23, 2020)
}

\begin{abstract}
The curing process of epoxy and amine mixtures was investigated from the viewpoint of molecular size of reactants via all-atom molecular dynamics simulation. Of four mixtures composed of either smaller or larger epoxy and amine molecules, the system of both smaller epoxy and amine reached the highest conversion at a given time, whereas the conversion for the combination of both larger epoxy and amine was the lowest. These can be explained in terms of their diffusion coefficients. In addition, the size effect on the reaction kinetics was more striking for epoxy than amine in the curing process. This was because epoxy reacted only once but amine did twice. When the primary amine reacted with epoxy, the resultant secondary amine was incorporated into the three-dimensional network. Hence, even if the primary amine had a higher mobility, the movement of the part after the reaction slowed down, making the further reaction to be the tertiary amine slow. Finally, it was shown that although the smaller epoxy reacted faster than the larger one in the mixed system of larger/smaller epoxy and amine, there was no difference in the reaction progress depending on the molecular size of amine.
\end{abstract}

Key Words: Curing reaction / Epoxy resin / Amine / Molecular size / Molecular dynamics

\section{INTRODUCTION}

Epoxy resins are widely used in a variety of applications from structural materials to modern devices due to their excellent thermal and mechanical properties ${ }^{1-3)}$. Also, since there is a degree of freedom in selecting epoxy as the base resin and amine as the curing agent, it is possible to select an appropriate mixture suitable for the desired properties by combining them, which is another factor for widening the application $^{4-12)}$. In addition, as further properties and/or functions are desired on to the epoxy resin, a number of components are supposed to be mixed in it.

In an epoxy resin composed of multi-components including various kinds of epoxy, the curing reaction with amine is competitive between/among various epoxy molecules. Similarly, when multiple amines are included, the reaction with epoxy becomes competitive as well. Hence, it is necessary to consider the reactivity of each epoxy with amine and vice-versa. In general, there are two factors influencing a chemical reaction such as the frequency factor and the activation energy. The molecular design for epoxy resins has been

\footnotetext{
$\dagger$ Corresponding author.

E-mail : s-yamamoto@cstf.kyushu-u.ac.jp (S.Y.); k-tanaka@cstf.kyushu-u.ac.jp (K.T.) Tel: +81-92-802-2877 (S.Y.); +81-92-802-2878 (K.T.)
}

extensively studied from the viewpoint of the activation ener$\mathrm{gy}^{13-16)}$. The activation energy of the reaction between epoxy and amino groups may change depending on the difference of adjacent functional group/s. In addition, an $\mathrm{OH}$ group in the reaction space accelerates the ring opening reaction of the epoxy group by hydrogen bond as a catalyst and also affects the reaction of the amino group ${ }^{13,15)}$. On the other hand, it seems to us that there is a lack of study dealing with the molecular design based on the frequency factors, namely the molecular mobility and the steric hindrance of reactants.

The kinetics of the network formation have been also widely studied for polymer systems ${ }^{17-21)}$. When the network formation evolves with the reaction progress, the apparent mobility of reactants decreases, making the reaction slow down ${ }^{19)}$. It seems reasonable to assume that this situation is basically the same as the curing reaction for epoxy resins. However, while the epoxy group reacts only once, the primary amino group reacts twice. This leads to be different from the simple network formation, especially in terms of the frequency factor. In fact, we previously found that when the curing process was simulated for a combination of epoxy and amine with different size, or different mobility, the reaction rate was dependent on the mobility only of the epoxy not the $\operatorname{amine}^{22)}$. 
In this study, the effect of molecular size on the curing process for epoxy resins was investigated via all-atom molecular dynamics (MD) simulations ${ }^{23-27)}$. Four systems were constructed using two kinds of larger and smaller molecules of epoxy and amine, and the progress of the curing reaction was analyzed. Further, the curing process was also studied in the system in which all four reactants were mixed.

\section{SIMULATIONS}

Using the all-atom MD simulation, the curing process of epoxy and amine, which proceeded in the two steps, was investigated. Figure 1 shows the reaction scheme of epoxy and amine. In the $1^{\text {st }}$ step shown in panel (a), an epoxy and a primary amine react to produce a secondary amine. Then, the resultant secondary amine reacts with the epoxy again to produce a tertiary amine in the $2^{\text {nd }}$ step shown in panel (b). Once the reaction occurs, the subsequent reaction is accelerated by the temperature increase because the above-mentioned two reactions are exothermic. In the MD simulations, the occurrence of the reaction was judged on the basis of the reaction probability $k$, which was defined as follows;

$$
k=A \cdot \exp \left(-\Delta E_{\mathrm{a}} / R T\right)
$$

where $A$ is an apparent frequency factor set as $1 \times 10^{8}$ so that the curing reaction is completed in a reasonable calculation time, $\Delta E_{\mathrm{a}}$ is the activation energy, $R$ is the gas constant and $T$ is the local absolute temperature at the reaction space ${ }^{23,26)}$. Given that a carbon atom of an epoxy group and a nitrogen atom of an amino group came close to a certain distance, if the $k$ value was larger than the generated random number of $0-1$, the reaction was allowed to proceed.

Although the $\Delta E_{\mathrm{a}}$ value depends on the combination of epoxy and amine, the current objective is to gain insight to the effect of molecular size. Hence, the values obtained experimentally in the previous studies were here used ${ }^{28-30)}$. That is, the values for the $1^{\text {st }}$ and $2^{\text {nd }}$ reactions were 56.8 and $55.3 \mathrm{~kJ} \cdot \mathrm{mol}^{-1}$, respectively. The heat generated by the reaction was taken into consideration by raising the kinetic energy of molecules involved in the reaction. The value of heat of (a)

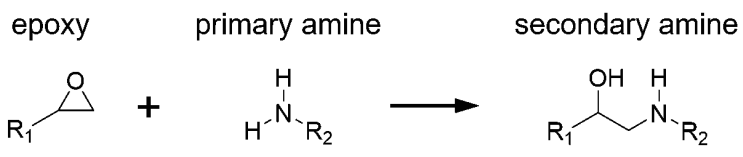

(b)

$$
\text { epoxy secondary amine tertiary amine }
$$

Fig. 1 Scheme of curing reaction process for epoxy and amine compounds; (a) $1^{\text {st }}$ step reaction of epoxy with primary amine to produce secondary amine and (b) $2^{\text {nd }}$ step reaction of epoxy with secondary amine to produce tertiary amine.

formation, $58.6 \mathrm{~kJ} \cdot \mathrm{mol}^{-1}$, which was obtained in previous experiments was set for the both reactions ${ }^{28-30)}$.

The distance at which the reaction possibly occurs has been often set to 0.4 to $0.6 \mathrm{~nm}^{23,26-28)}$. However, the effect of the reaction distance on the progress of curing process seems to be not discussed. Thus, before starting the simulation in this study, the reaction distance dependence was examined for the system consisting of hydrogenated bisphenol-A diglycidyl ether (HDGEBA) and 1,4-cyclohexanebis(methylamine) (CBMA). Figure 2 shows the time course of reaction conversion for various reaction distances. The conversion increased with increasing reaction distance from 0.4 to $0.6 \mathrm{~nm}$ at a given time. However, if a larger value is adopted, the reaction may occur in an unrealistic arrangement due to the steric hindrance. This implies that the distance is better as small as possible. On the other hand, if the distance between epoxy and amine is too close such as $0.3 \mathrm{~nm}$, almost no reaction occurs. Taking into the above accounts, we decided to use $0.4 \mathrm{~nm}$ as the reaction distance in this study.

Figure 3 shows the chemical structures of epoxy and amine with different sizes. The larger molecules are bisphenol A type epoxy (Ep-L) and amine (Am-L), and the smaller ones are linear chain type epoxy (Ep-S) and amine (Am-S). Table I collects characteristic properties of these molecules. The molecular volume was measured by scanning the surface with a water molecule size probe. The larger molecules are almost twice as large as the smaller ones. Density and self-diffusion coefficients were calculated via MD simulations for a

Table I Characteristic properties of larger/smaller epoxy and amine molecules.

\begin{tabular}{ccccc}
\hline epoxy-amine & molecular weight & $\begin{array}{c}\text { molecular volume } \\
\left(\mathrm{nm}^{3}\right)\end{array}$ & $\begin{array}{c}\text { density } \\
\left(\mathrm{g} \cdot \mathrm{cm}^{-3}\right)\end{array}$ & $\begin{array}{c}\text { self-diffusion coefficient } \\
\left(10^{-10} \mathrm{~m}^{2} \cdot \mathrm{s}^{-1}\right)\end{array}$ \\
\hline Ep-L & 340 & 0.344 & 1.14 & 0.14 \\
Ep-S & 174 & 0.179 & 1.12 & 2.00 \\
Am-L & 310 & 0.359 & 1.01 & 0.16 \\
Am-S & 144 & 0.176 & 0.86 & 2.46 \\
\hline
\end{tabular}




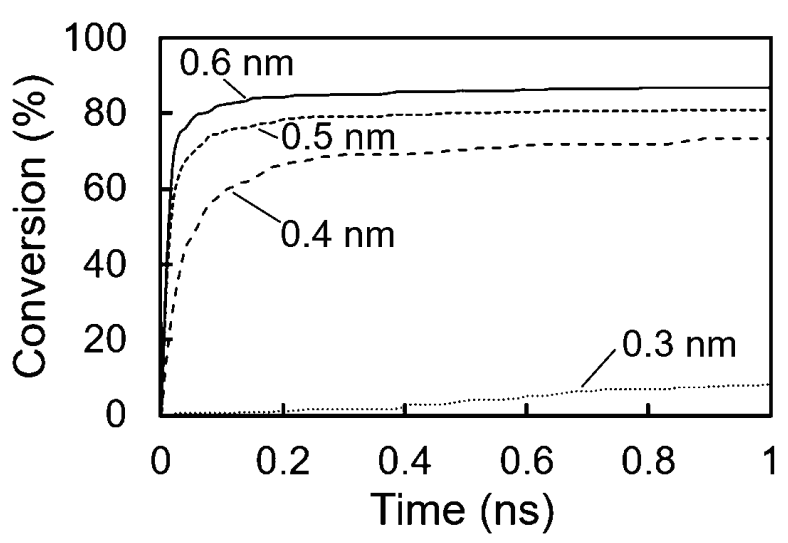

Fig. 2 Effect of reaction distance on conversion for the system consisting of HDGEBA and CBMA.

(a)

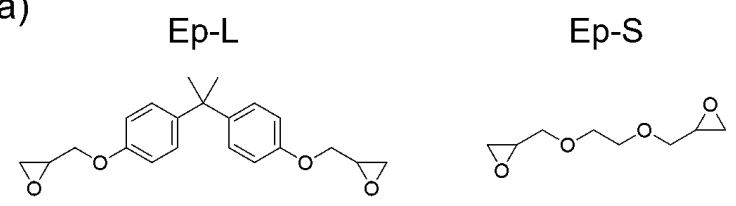

(b)

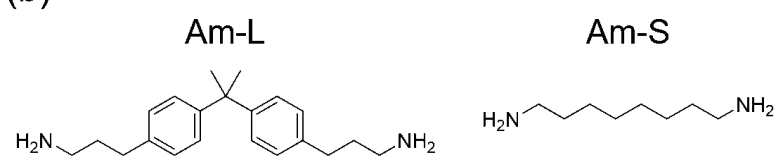

Fig. 3 Chemical structures of larger and smaller molecules of (a) epoxy and (b) amine.

system consisting of 200 molecules at $296 \mathrm{~K}$ and $1 \mathrm{~atm}$, obtained by averaging for three different initial structures.

MD simulations were performed using the Materials Studio 2020 (Dassault Systémes). Using the Amorphous Cell module, 100 epoxy and 50 amine molecules in stoichiometry were randomly packed into a cubic unit cell. Then, an equilibrium density was calculated by MD simulation at $296 \mathrm{~K}$ and $1 \mathrm{~atm}$ with COMPASS II forcefield ${ }^{31,32)}$ and NPT ensemble. The Nosé-Hoover-Langevin (NHT) algorithm and the Berendsen method were used for temperature and pressure controls, respectively. For the summation method of electrostatic interaction, the Particle-Particle Particle-Mesh (PPPM) algorithm ${ }^{33)}$ was adopted. The atom-based summation method was used for van der Waals term with a cutoff distance of $1.25 \mathrm{~nm}$. Next, the curing process for each model was simulated for $10 \mathrm{~ns}$ by the procedure described above. In our previous study, the NVT ensemble was used in consideration of computing efficiency, but here we compared it with NPT ensemble. Finally, the curing process was calculated in the same manner as above, using a system in which all epoxies and amines were mixed, there are 100 for Ep-L and Ep-S and 50 for Am-L and Am-S, respectively. All calculations were performed on three different initial structures and the results so obtained were averaged over them.

\section{RESULTS AND DISCUSSION}

Figure 4 shows snapshots of the cross-linking network structure evolved in the Ep-L/Am-S system. The side length of a cubic cell is $4.0 \mathrm{~nm}$. Each color represents a fragment connected by a chemical bond. In the initial state shown in panel (a), there were 150 molecules of unreacted epoxy and amine, so there were 150 colors. The reaction conversion was defined by the consumption ratio of epoxy molecules. The transition of colors from panels (a) to (f) shows how the reaction proceeded. The number of colors decreased, meaning that the reaction progressed and the fragments grew and coalesced. A large blue fragment appeared around the reaction conversion of 60 to $70 \%$. This makes it clear that the network structure was percolated through the entire system, and is consistent with the commonly-reported gel point of $60-70 \%$ for epoxy resins ${ }^{34,35)}$. The curing reaction proceeded in the same manner in other epoxy/amine combination systems.

Figure 5 (a) shows the time course of the reaction conversion for all four systems using the NPT ensemble. As a general trend, the reaction proceeded rapidly up to $0.2 \mathrm{~ns}$ and then slowed down. The final reaction conversion at $10 \mathrm{~ns}$ was larger in the order of Ep-S/Am-S > Ep-S/Am-L > Ep-L/Am-S $>$ Ep-L/Am-L. For comparison, the results using the NVT ensemble are shown in panel (b). The progress of the curing reaction, the final conversion, and the order thereof were in (a)

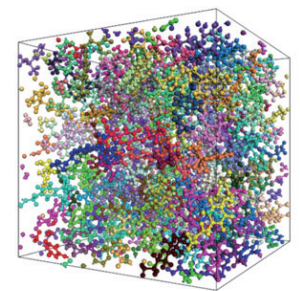

(c)

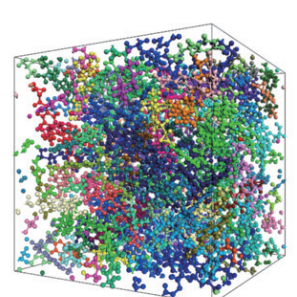

(e)

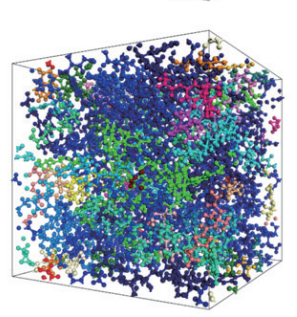

(b)

(d)

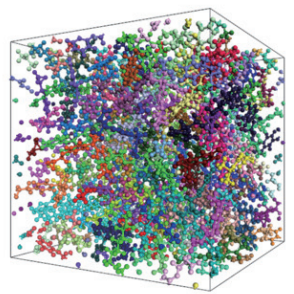

(f)
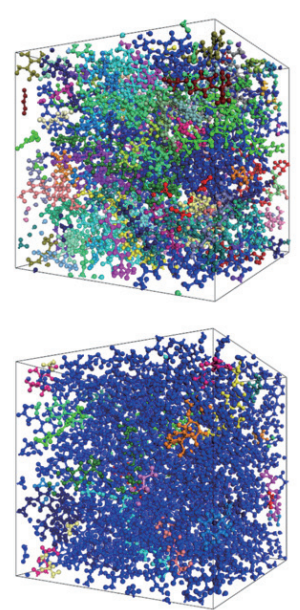

Fig. 4 Snapshots of the Ep-L/Am-S system during the curing process at the reaction conversion of (a) $0 \%$, (b) $20 \%$, (c) $40 \%$, (d) $50 \%$, (e) $60 \%$, and (f) $70 \%$. Different colors are displayed for each fragment connected by chemical bond. 

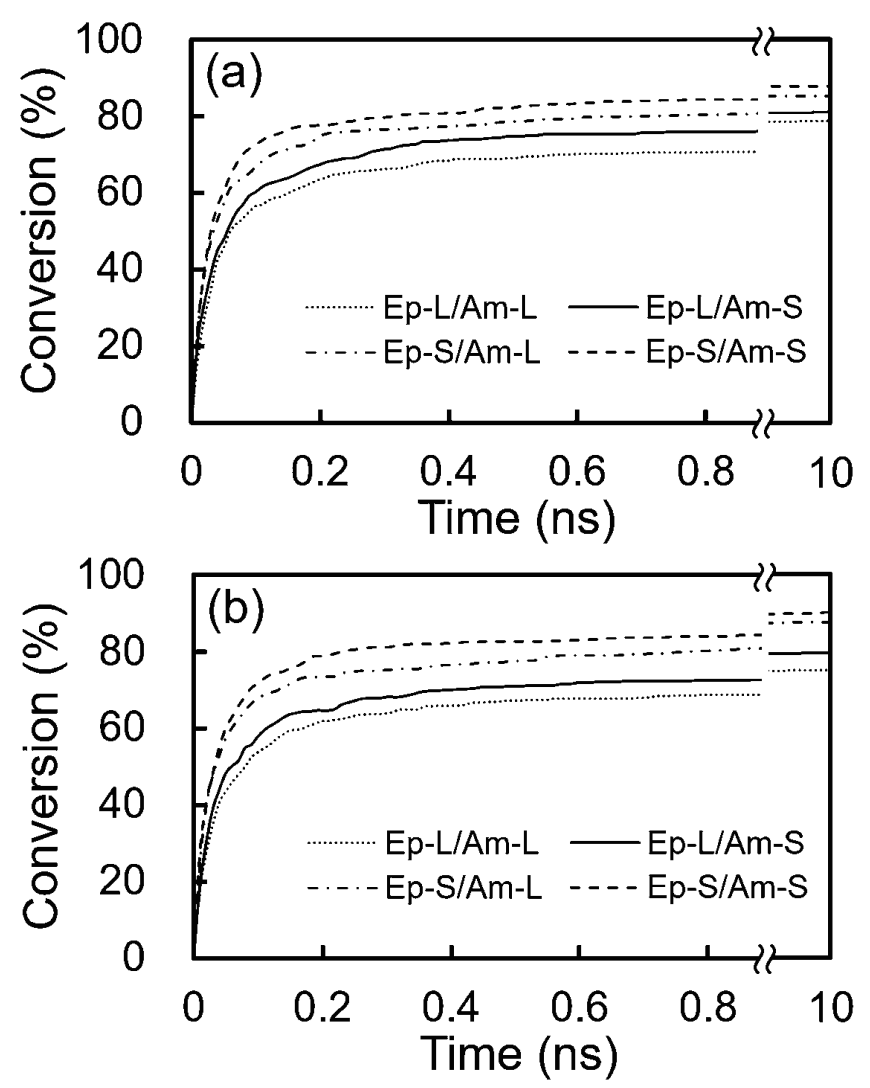

Fig. 5 Time evolution of reaction conversion using (a) NPT and (b) NVT ensembles. The conversion is defined as the consumption ratio of epoxy groups.

good agreement with those obtained using the NPT ensemble shown in panel (a). Considering these results, there is nothing wrong with the use of the NVT ensemble for the analysis of reaction processes. Of course, since the density increased due to the curing shrinkage as described later, it was necessary to simulate with the NPT ensemble so that the mechanical properties of the cured product could be discussed.

Table II summarizes the density before and after the curing reaction, the increase rate in density, and the final reaction conversion. While the initial density depended on the combination of epoxy and amine, the density after the curing was almost the same among the four systems. The curing reaction made the density of the resins larger by 2 to $10 \%$. This is in good accordance with the curing shrinkage of the epoxy resin experimentally observed ${ }^{36)}$.

Let us discuss the reaction progress depending on the molecular size of reactants. The reaction between epoxy and amine occurs when they come close to each other. Since the activation energy of the reaction was set to be the same in all combinations, it is apparent that the frequency which epoxy met amine should become dominant to determine the reaction progress. Hence, the molecular size effect on the reaction progress can be discussed on the basis of the self-diffusion coefficients $D$ of the reactants. Table III shows $D$ values of epoxy and amine molecules from the MD simulation using the NPT ensemble for $1 \mathrm{~ns}$ at $296 \mathrm{~K}$. In the case of the Ep-S/ Am-S system, which was the combination of smaller epoxy and amine molecules before the curing reaction, the both had the largest $D$ values of each. Considering this, it can be easily understood that the reaction proceeded fastest in this system. On the contrary, in the Ep-L/Am-L system composed of larger epoxy and amine molecules, their diffusion coefficients were the smallest of each, resulting in the slowest curing reaction. One point should be here addressed is that the number density of functional groups was also a function of the molecular size. The difference between smaller and larger molecules was approximately twice. Taking into account a fact that the diffusion was faster for smaller molecules than for larger one by $c a$. twenty, the concentration effect of functional groups on the reaction kinetics is trivial if any.

For the two "asymmetric" systems in terms of the reactant size, namely Ep-L/Am-S and Ep-S/Am-L, each smaller molecule had a larger diffusion coefficient. Also, Ep-S and Am-S alone had the similar mobility. However, the mobility was much higher for the Ep-S/Am-L system than for the Ep-L/Am-S, as shown in Table III. Taking into account that the fractional amount of epoxy molecules, which moved faster due to its size, was larger than that of amine due to stoichiometric ratio, the mobility difference between the Ep-S/ Am-L and Ep-L/Am-S systems could be understood. In addition, there is another explanation. As discussed before, a primary amine became a secondary amine in the $1^{\text {st }}$ step reaction, and was incorporated into the reaction product. Consequently, the mobility of the secondary amine was supposed to be reduced. This was not the case for epoxy because it reacted only once.

Figure 6 shows the time evolution, from (a) to (d), of a representative fragment linked by chemical bonds in the

Table II Density before and after the curing reaction and the increase rate in density with the final conversion at $10 \mathrm{~ns}$.

\begin{tabular}{ccccc}
\hline epoxy-amine & $\begin{array}{c}\text { initial density } \\
\left(\mathrm{g} \cdot \mathrm{cm}^{-3}\right)\end{array}$ & $\begin{array}{c}\text { final density } \\
\left(\mathrm{g} \cdot \mathrm{cm}^{-3}\right)\end{array}$ & increase rate (\%) & conversion (\%) \\
\hline Ep-L/Am-L & 1.09 & 1.12 & 2.9 & 78.5 \\
Ep-L/Am-S & 1.08 & 1.12 & 3.5 & 81.0 \\
Ep-S/Am-L & 1.06 & 1.13 & 5.8 & 85.0 \\
Ep-S/Am-S & 1.02 & 1.12 & 9.6 & 87.7 \\
\hline
\end{tabular}


Table III Self-diffusion coefficients $D$ of epoxy and amine molecules before the curing reaction.

\begin{tabular}{ccc}
\hline epoxy-amine & $D_{\text {epoxy }}\left(10^{-10} \mathrm{~m}^{2} \cdot \mathrm{s}^{-1}\right)$ & $D_{\text {amine }}\left(10^{-10} \mathrm{~m}^{2} \cdot \mathrm{s}^{-1}\right)$ \\
\hline Ep-L/Am-L & 0.14 & 0.15 \\
Ep-L/Am-S & 0.18 & 0.43 \\
Ep-S/Am-L & 0.84 & 0.48 \\
Ep-S/Am-S & 2.28 & 2.56 \\
\hline
\end{tabular}

(a)

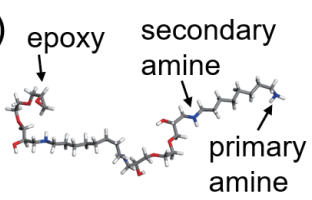

(c)

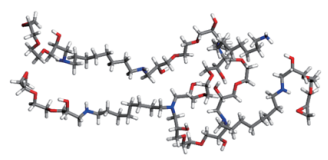

(b)

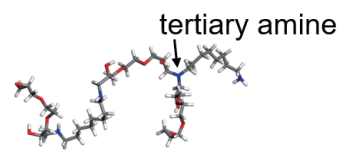

(d)

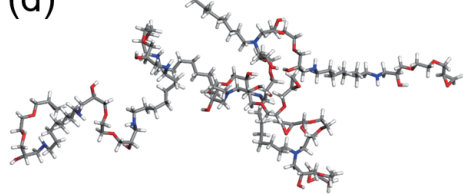

Fig. 6 Representative snapshots of fragments linked by chemical bonds in the Ep-S/Am-S system at conversions of (a) $10 \%$, (b) $20 \%$, (c) $30 \%$, and (d) $40 \%$. Carbon, oxygen, nitrogen, and hydrogen atoms are colored gray, red, blue, and white, respectively.

Ep-S/Am-S system. Once a molecule is incorporated into the product, the mobility is reduced. In the case of amine, a primary amine group reacts with an epoxy group to be a secondary amine, which reacts with an epoxy group once more. Thus, the mobility reduction should be much more serious for amine.

To confirm this idea, the self-diffusion coefficients $D$ of epoxy and amine groups during the curing reaction were examined. Figure 7 shows $D$ for epoxy and primary, secondary and tertiary amine groups as a function of conversion for the Ep-S/Am-S system. Unreacted epoxy and primary amine had larger $D$ values. Also, the value became approximately one third for secondary amine. Even if the mobility of the primary amine was high, the secondary amine produced after the $1^{\text {st }}$ step reaction became slower. This is the reason why the reaction progress was more affected by the mobility of epoxy rather than amine. Considering this, it can be claimed that the mobility of epoxy is of pivotal importance to design the curing process for the epoxy resin.

Finally, the curing process was calculated for the mixture composed of all kinds of epoxy and amine. The progress of the curing reaction was similar to that of each combination shown in Fig. 5 (a), and the final reaction conversion was $80.6 \%$. Figure 8 shows the time dependence of residual epoxy as well as primary and secondary amino groups in the Ep-L/Ep-S/Am-L/Am-S system. Epoxy and primary amino groups were stoichiometrically consumed all at once from the beginning, producing concurrently secondary amine

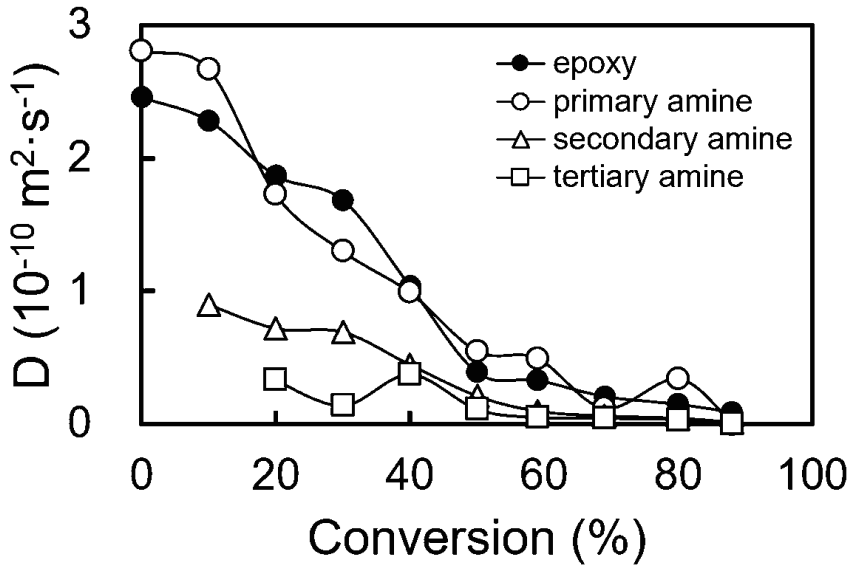

Fig. 7 Self-diffusion constants for each functional group as a function of reaction conversion for the Ep-S/Am-S system.

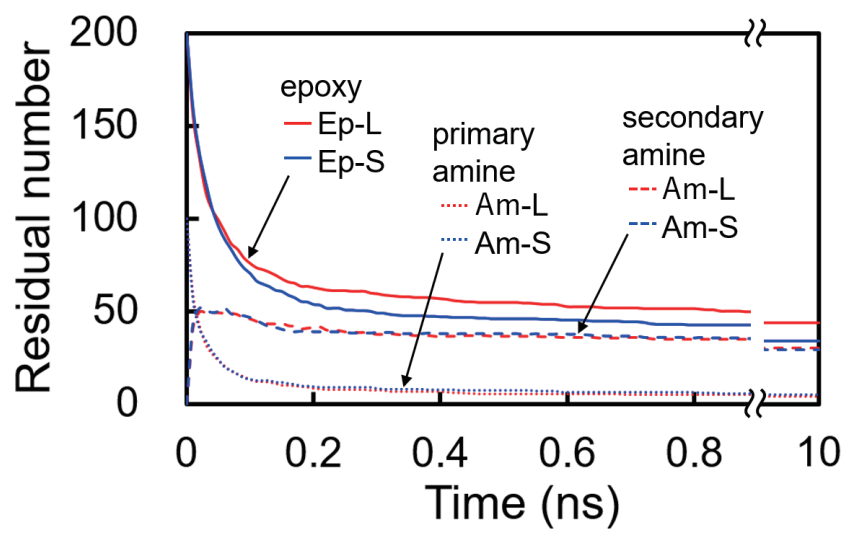

Fig. 8 Time course of residual functional groups in the Ep-L/Ep-S/Am-L/ Am-S system.

groups. Focusing on epoxy groups, smaller molecules, Ep-S, were consumed faster than Ep-L due to the higher mobility. On the other hand, the size effect on the consumption rate was not clear for amines. This trend was consistent with the mechanism described above. Therefore, again, it can be claimed that the effect of epoxy mobility is more striking in the curing process.

\section{CONCLUSIONS}

The curing reaction was analyzed for the combination of epoxy and amine having different sizes via all-atom MD simulations. Setting the activation energy of the curing reactions to be the same in all cases, the effect of molecular size on the reactions was discussed. The mixture composed of both smaller epoxy and amine had the fastest reaction progress thanks to the largest frequency induced by the highest molecular mobility. On the contrary, the system in which larger reactants were mixed had the slowest reaction progress. Comparing the reaction for the mixtures composed of larger epoxy and smaller amine and vice-versa, it proceeded faster with the smaller epoxy than with the smaller amine. This was 
because the epoxy and primary amino groups reacted only once and twice, respectively. No matter how high the mobility of a primary amine was, a secondary amine which was the product from the primary one was incorporated into the product, the network, so that its mobility decreased and the subsequent reaction to a tertiary amine slowed down. In the system in which four kinds of larger and smaller epoxy and amine were mixed all together, the smaller epoxy reacted faster than others. Therefore, it can be concluded that the molecular sizes of epoxy and amine are very much and less important to the kinetic of the curing reaction, respectively.

\section{ACKNOWLEDGMENTS}

This research was supported by JST-Mirai Program (JPMJMI18A2). The authors wish to thank R. Kuwahara (Dassault Systèmes K. K.) for helpful comments and discussions.

\section{REFERENCES}

1) Liu S, Chevali VS, Xu Z, Hui D, Wang H, Compos B Eng, 136, 197 (2018).

2) Aoki R, Yamaguchi A, Hashimoto T, Urushisaki M, Sakaguchi T, Kawabe K, Kondo K, Iyo H, Polym J, 51, 909 (2019).

3) Bach Q-V, Vu CM, Vu HT, Hoang T, Dieu TV, Nguyen DD, Polym J, 52, 345 (2020).

4) Ishida H, Allen DJ, J Polym Sci B, 34, 1019 (1996).

5) Knorr Jr DB, Yu HY, Richardson AD, Hindenlang MD, McAninch IM, La Scala JJ, Lenhart JL, Polymer, 53, 5917 (2012).

6) Pramanik M, Fowler EW, Rawlins JW, Polym Eng Sci, 54, 1990 (2014).

7) Liu F, Wu L, Song Y, Xia W, Guo K, RSC Adv, 5, 45987 (2015).

8) Gao L, Zhang Q, Li H, Yu S, Zhong W, Sui G, Yang X, Polym Chem, 8, 2016 (2017).

9) Livi S, Chardin C, Lins LC, Halawani N, Pruvost S, DuchetRumeau J, Gérard J-F, Baudoux J, ACS Sustainable Chem Eng, 7, 3602 (2019).

10) Abdollahi H, Salimi A, Barikani M, Samadi A, Hosseini Rad S, Zanjanijan AR, J Appl Polym Sci, 136, 47121 (2019).

11) Russel BK, Takeda S, Ward C, Hamerton I, React Funct Polym, 144, 104353 (2019).
12) Hubbard RL, Tyler DR, Thompson B, J Appl Polym Sci, e49635 (2020).

13) Jouyandeh $M$, Shabanian $M$, Khaleghi $M$, Paran SMR, Ghiyasi S, Vahabi H, Formela K, Puglia D, Saeb MR, Prog Org Coat, 125, 384 (2018).

14) Estridge CE, Polymer, 141, 12 (2018).

15) Mora A-S, Tayouo R, Boutevin B, David G, Caillol S, Eur Polym J, 123, 109460 (2020).

16) Huang C, Sun $X$, Yuan H, Song C, Meng Y, Li X, Vib Spectrosc, 106, 102993 (2020).

17) Norrish RGW, Smith RR, Nature, 150, 336 (1942).

18) Russell GT, Gilbert RG, Napper DH, Macromolecules, 25, 2459 (1992).

19) Achilias DS, Macromol Theory Simul, 16, 319 (2007).

20) Lang M, John A, Sommer J-U, Polymer, 82, 138 (2016).

21) Masubuchi Y, Uneyama T, Soft Matter, 15, 5109 (2019).

22) Yamamoto S, Tanaka K, Soft Matter, 17, 1359 (2021).

23) Okabe $T$, Takehara $T$, Inose $K$, Hirano $N$, Nishikawa $M$, Uehara T, Polymer, 54, 4660 (2013).

24) Yang Q, Li X, Shi L, Yang X, Sui G, Polymer, 54, 6447 (2013).

25) Hamerton I, Tang W, Anguita JV, Silva SRP, Reac Funct Polym, 74, 1 (2014).

26) Okabe T, Oya Y, Tanabe K, Eur Polym J, 80, 78 (2016).

27) Yamamoto S, Kuwahara R, Aoki M, Shundo A, Tanaka K, ACS Appl Polym Mater, 2, 1474 (2020).

28) Aoki M, Shundo A, Kuwahara R, Yamamoto S, Tanaka K, Macromolcules, 52, 2075 (2019).

29) Aoki M, Shundo A, Okamoto K, Ganbe T, Tanaka K, Polym J, 51, 359 (2019).

30) Aoki M, Shundo A, Yamamoto S, Tanaka K, Soft Matter, 16, 7470 (2020).

31) Sun H, Jin $Z$, Yang C, Akkermans RLC, Robertson SH, Spenley NA, Miller S, Todd SM, J Mol Model, 22, 1 (2016).

32) Sun H, J Phys Chem B, 102, 7338 (1998).

33) Hockney RW, Eastwood JW, Computing Simulation using Particles, Adam-Hilger, Bristol, (1988).

34) Tanaka Y, Stanford JL, Stepto R, Macromolecules, 45, 7197 (2012).

35) Zhu Z, Sun X, Yuan H, Song C, Cao Y, Li X, Polym Test, 72, 416 (2018).

36) Minty RF, Thomason JL, Yang L, Stanley W, Roy A, Polym Test, 73, 316 (2019). 